Georgia State University

ScholarWorks @ Georgia State University

\title{
Childhood Healthcare Experience, Healthcare Attitudes, and Optimism as Predictors of Adolescents' Healthcare Behavior
}

Tina Jones

Melissa DeMore

Lindsey L. Cohen

Georgia State University, Ilcohen@gsu.edu

Cara O'Connell

Deborah Jones

Follow this and additional works at: https://scholarworks.gsu.edu/psych_facpub

\section{Recommended Citation}

Jones, T., DeMore, M., Cohen, L. L., O’Connell, C., \& Jones, D. J. (2008). Childhood healthcare experience, healthcare attitudes, and optimism as predictors of adolescents' healthcare behavior. Journal of Clinical Psychology in Medical Settings, 15(3), 234-240. DOI: 10.1007/s10880-008-9126-7

This Article is brought to you for free and open access by the Department of Psychology at ScholarWorks @ Georgia State University. It has been accepted for inclusion in Psychology Faculty Publications by an authorized administrator of ScholarWorks @ Georgia State University. For more information, please contact scholarworks@gsu.edu. 
Running head: PREDICTORS OF HEALTHCARE BEHAVIOR

Childhood Healthcare Experience, Healthcare Attitudes, and Optimism as Predictors of Adolescents' Healthcare Behavior

\author{
Tina Jones ${ }^{1}$ \\ Melissa DeMore ${ }^{2}$ \\ Lindsey L. Cohen ${ }^{3}$ \\ Cara O’Connell ${ }^{4}$ \\ Deborah Jones 5
}

1. Department of Psychology, West Virginia University, Morgantown, WV

2. Department of Behavioral Psychology, The Kennedy Krieger Institute, Johns Hopkins University School of Medicine, Baltimore, MD

3. Department of Psychology, Georgia State University, Atlanta, GA

Corresponding author: Lindsey L. Cohen, Ph.D. Department of Psychology, Georgia State University, Atlanta, GA 30302-5010

304-413-6263; 1lcohen@gsu.edu

4. Department of Rehabilitation, University of North Carolina, Chapel Hill, Chapel Hill, NC

5. Department of Psychology, University of North Carolina, Chapel Hill, Chapel Hill, NC 


\begin{abstract}
Late adolescence and early adulthood is a time when people establish many of their life-style behaviors. Thus, in order to promote optimal health, it is important to identify factors predictive of young adults' healthcare behavior. This study evaluated the relationship between measures of childhood healthcare experience, healthcare attitudes, and optimism with young adults' healthcare behavior in a sample of college students $(n=100)$. Results suggested that prior healthcare experience, attitudes about healthcare, and optimism are associated with current healthcare behavior. In addition, the relation between childhood healthcare experience and current healthcare behavior was moderated by optimism, such that those who reported both more negative childhood healthcare experiences and low levels of optimism reported the least adaptive healthcare behaviors and those who reported the most positive childhood healthcare experience and the highest levels of optimism reported the most adaptive healthcare behavior.
\end{abstract}

Key words: Adolescence; Healthcare; Optimism; Healthcare experience 
Childhood Healthcare Experience, Healthcare Attitudes, and Optimism as Predictors of Adolescents' Healthcare Behavior

Late adolescence is a time of transition when lifestyle behaviors are developing (Feldman, 1998; Martinelli, 1999). Unfortunately, many college students engage in a variety of unhealthy behaviors (e.g., excessive alcohol consumption, smoking) and do not perform adaptive healthcare behaviors (e.g., regular medical checkups, proper diet and exercise; Grace, 1997; Sax, 1997). Identifying and understanding variables related to adolescents' healthcare practices should aid in the development of interventions to promote healthy behavior.

Theoretical models might help guide and organize variables related to adolescent healthcare behavior. Becker $(1977,1990)$ proposed the Health Belief Model (HBM), which posits that personal cognitions influence healthcare behaviors. The HBM is predictive of healthcare behaviors in late adolescents (for review, see Janz \& Becker, 1984; Reyna \& Farley, 2006) and has been identified as a model for health promotion and behavior change that is developmentally appropriate for application to adolescents (Pan American Health Organization, 2005).

It is likely that early healthcare experiences influence future beliefs about the value of healthcare. In fact, childhood healthcare experiences have been found to predict healthcare attitudes and behaviors. For example, Hackworth and McMahon (1991) found that children who reported more pain also reported less satisfaction with and more avoidance of healthcare. Additionally, memories of stressful and painful medical experiences from childhood have been shown to be positively associated with medical fear and pain as an adolescent (Pate et al., 1996). This pain and fear as a child might contribute to adulthood avoidance of medical situations (Pate 
et al., 1996). It has also been suggested that child medical stress is positively correlated with avoidance of various medical staff and hospitals (Bachanas \& Roberts, 1995).

Conversely, positive early healthcare experiences have been associated with later positive healthcare behaviors. For example, Pate et al. (1996) found increased effective coping as a child predicted decreased college student pain, fear, and avoidance. Furthermore, it has been conjectured that positive healthcare experiences in childhood may render children more likely to adopt positive or health-promoting attitudes towards healthcare and subsequently seek out medical care when appropriate (Hackworth \& McMahon, 1991; Bachanas \& Roberts, 1995). Indeed, increased positive contact with healthcare professionals has been associated with more positive attitudes (Peterson, Ross, \& Tucker, 2002).

In addition to early experiences, an individual's attitudes (i.e., their feelings, emotions, predispositions, impressions, and opinions) about healthcare are linked to their healthcare behavior. For example, negative healthcare attitudes increased the likelihood of engaging in negative healthcare behaviors (i.e., avoidance of medical visits, fear of medical procedures; Pate et al., 1996; Bachanas \& Roberts, 1995; Peterson et al., 2002). Similarly, positive attitudes toward condoms have been associated with better adherence to condom use (Morrill, Ickovics, Golubchikov, \& Beren, 1996). However, there is a lack of research on the relationships between healthcare attitudes and other specific positive healthcare behaviors (e.g., diet, exercise).

Optimism is another factor that may impact an individual's engagement in preventive healthcare. Optimism can be defined as the expectation that one will experience more good than bad outcomes (Scheier \& Carver, 1992). Optimism has been found to predict many mental and physical health outcomes (e.g., positive mood, good health, effective problem solving, and long life; Peterson, 2000). Research has also found that optimists engage in more health promoting 
behaviors (Morill et al., 1996; Scheier \& Carver; Segerstrom, Taylor, Kemeny, \& Fahey, 1998), as compared to pessimistic individuals.

Optimism has also been linked to healthcare behaviors such as condom use (Morill et al., 1996) and maintenance of a balanced diet and regular exercise (Morill et al., 1996; Scheier \& Carver, 1992; Segerstrom et al., 1998). Although much is known about optimism and health status, there is still a need to examine possible relations between optimism and other healthcare behaviors (e.g., personal hygiene, tobacco use, attendance at annual physician well check-ups).

In sum, adolescents are in a transition stage wherein healthy (e.g., regular medical care) or unhealthy (e.g., smoking cigarettes) behaviors develop. Although research has indicated that childhood healthcare experiences (Pate et al., 1996), healthcare attitudes (Ajzen \& Fishbein, 1977), and optimism (Peterson, 2000) are each related to late adolescent health practices, an extensive literature search found no study that has attempted to examine whether the variables might be interrelated to further explain adolescents' healthcare behavior. The identification of predictors of adolescents' healthcare practices should aid in the development of interventions to promote healthy behavior.

Given the lack of appropriate measures of prior healthcare experience and current healthcare behavior, one aim of the current study was to create and validate scales of these two constructs. However, the primary aim was to investigate how childhood healthcare experience, healthcare attitudes, and optimism might each predict current adult healthcare behavior in late adolescents or young adults. It was hypothesized positive childhood healthcare experience, adaptive healthcare attitudes, and optimism would be positively correlated with more adaptive healthcare behaviors, whereas negative childhood healthcare experiences, poor attitudes, and pessimism would predict maladaptive healthcare behavior. A second primary aim of our study 
was to examine if the hypothesized relationship between childhood healthcare experiences and current healthcare behaviors were moderated by optimism (see figure 1) or healthcare attitudes (see figure 2) each, as might be predicted by the HBM.

Method

\section{Participants}

Participants were 100 undergraduate introductory to psychology students (76 females) at a university located in a rural state in the northeastern U.S. Participants were predominately Caucasian $(89 \%)$, individuals aged 18 to 23 years $(M=18.57$ years, $S D=.81$ years $)$. The majority of participants reported no history of chronic illness $(85 \%)$ or serious illness $(78.9 \%)$ and most (92\%) had health insurance. Moreover, most participants (77\%) indicated that they had made 1-3 doctors' visits in the past year. Table 1 includes a summary of the demographic variables for the sample.

\section{Measures}

Childhood Healthcare Experience Inventory (CHEI). The CHEI was developed for this study. Based on the Medical Experience Questionnaire (MEQ; Pate et al., 1996), the CHEI is a 32-item paper-and-pencil questionnaire designed to assess adolescents' recollections of their childhood healthcare experiences. For example, items include the following: "As a child, how afraid were you to go to the doctor?", "Did you like your doctor?", "How afraid were you of getting an injection.” All questions were answered using visual analogue scales (VASs) consisting of $100 \mathrm{~mm}$ lines with anchors such as "not at all" to "very." Total scores range from 0 - 3200 with higher total scores reflecting positive childhood healthcare experiences. The CHEI showed good internal consistency $(\alpha=.88)$ with this sample. 
Healthcare Behaviors Inventory (HBI). The HBI was created for this study and was also based on the MEQ (Pate et al., 1996). Whereas the MEQ queried about general impressions of health, the HBI assessed specific healthcare behavior (e.g., attending checkups). The HBI is a 42-item questionnaire that queries participants about their current healthcare behavior. For example, items include "Do you wash your hands before eating?", "Do you smoke cigarettes?", and "Do you see your doctor regularly?" Questions are answered using visual analogue scale (VASs) consisting of $100 \mathrm{~mm}$ lines with anchors such as, "never" to "always." The HBI ranges from $0-4200$ with higher total scores indicating positive current healthcare behavior. The HBI showed good internal consistency $(\alpha=.86)$ in the current study.

Health Perceptions Questionnaire and General Health Rating Index (HPQ; Ware, 1976). Ware designed the HPQ to assess perceptions of health status. The measure was used in the current study to validate both the CHEI and HBI. The 32-item questionnaire has eight subscales. The scale demonstrated good internal consistency in the current study $(\alpha=.87)$. The Prior Health subscale was used to examine content validity of the CHEI, and the Current Health and Attitude toward going to the Doctor subscales were used to validate the HBI.

Healthcare Attitudes Questionnaire (HCAQ; Hackworth \& McMahon, 1991). The HCAQ is a modified version of the Children's Healthcare Attitudes Questionnaire (CHAQ; Bush $\&$ Holmbeck, 1987). The healthcare attitudes questionnaire contains 24 items, such as "Do you like to go to the doctor?" Response choices range from "I really hate them" to "I really like them". Summed scores on the attitude scales range from 0-96 with higher scores indicating more positive healthcare attitudes, and summed scores on the pain scales range from 0-68 with higher scores indicating greater perceptions of pain. The total attitude score on the questionnaire was used in analyses. Prior research has indicated that the HCAQ has adequate reliability with 
Cronbach's alpha coefficients ranging from .63 to .86 on the attitude scale and from .69 to .84 on the pain scale (see Hackworth and McMahon). In the current study, Cronbach's alpha for the attitude scale was .83 .

Life Orientation Test (LOT; Scheier \& Carver, 1994). The LOT is compromised of eight coded items; four items are used to measure optimism and four to measure pessimism. Questions are posed to tap optimism, "In certain times, I usually expect the best," and pessimism, "I hardly ever expect things to go my way." The responses are rated from "Strongly disagree" (0) to "Strongly agree" (4) on a 5-point Likert scale. The items are summed to give a total score with higher scores indicating greater optimism. Prior research has suggested that the LOT has good internal consistency with Cronbach's alpha .82 (Scheier \& Carver, 1994) In the current sample the LOT also shown good internal consistenc $(\alpha=.88)$.

\section{Procedures}

This study received the approval of the West Virginia University Institutional Review Board (IRB). Participants were recruited by placing advertisements in the psychology building and presenting information about the study in the introductory to psychology classes. Interested students set up appointments or attended pre-arranged appointments with researchers. The researcher explained the study, presented consent forms and study questionnaires, and answered any questions. It took approximately one hour for students to complete all the measures. Participants received extra credit points in their psychology course for participation.

$$
\text { Results }
$$

\section{Descriptive statistics}

Descriptive statistics were used to summarize the study variables (Table 1) and study variables were found to be correlated (Table 2). One-way ANOVAs indicated no significant 
differences across ethnicity or gender on the CHEI, HCAQ, LOT, or HBI. Further, Pearson product-moment correlations suggested no significant relations between age and the CHEI, HCAQ, LOT, and HBI summary scores.

\section{CHEI and HBI Content Validity}

In the assessment of the CHEI and HBI content validity, Pearson's product-moment correlations indicated significant relations between the CHEI and the HPQ Prior Health subscale $(r=.25, p<.01)$, the HBI and the HPQ Current Health subscale $(r=.27, p<.01)$, and the HBI and HPQ Attitude toward going to the Doctor subscale $(r=.46, p<.01)$.

\section{Moderation}

To evaluate whether healthcare attitudes or optimism moderated the relation between childhood healthcare experience and current healthcare behavior (Figures 1 and 2), hierarchical regression analyses were conducted (Tables 3 and 4). In the first block, the main effects of the proposed independent variable, childhood healthcare experience, and the respective moderator variable (attitudes or optimism), were entered. In the second block, the interaction of childhood healthcare experience with the respective moderator was entered (childhood healthcare experience $\mathrm{x}$ attitudes or childhood healthcare experience $\mathrm{x}$ optimism). Consistent with the recommendations of Baron and Kenny (1986) the variables were centered prior to creating the interaction term to reduce multicollinearity. Regression analyses were run separately for each of the proposed moderator variables.

In regression analyses, childhood healthcare experiences were associated with adolescent healthcare attitudes, $\beta=.69, \mathrm{p}<.01$ (Table 3). More favorable childhood healthcare experiences were associated with more adaptive adolescent healthcare behaviors. Adolescent healthcare attitudes were also associated with adolescent health behaviors, $\beta=.23, \mathrm{p}<.01$, such that more 
adaptive healthcare attitudes were associated with more adaptive healthcare behaviors.

Healthcare attitudes, however, did not moderate the association between childhood healthcare experiences and adolescent healthcare behaviors, $\beta=-.92$, n.s.

As predicted, the LOT was also associated with adolescent healthcare behaviors, $\beta=.17$, $\mathrm{p}<.05$ (Table 4). Higher levels of optimism were associated with more adaptive healthcare behaviors. Additionally, the LOT moderated the association between the CHEI and adolescent healthcare behaviors, $\beta=.91, \mathrm{p}<.05$. The interaction was explicated by conducting a median split on the LOT and examining the association between the CHEI and adolescent healthcare behaviors at each level. As demonstrated in Figure 3, those who reported both more negative CHEI and low levels of optimism reported the least adaptive healthcare behaviors and those who reported the most positive $\mathrm{CHEI}$ and the highest levels of the LOT reported the most adaptive healthcare behavior.

\section{Discussion}

Initial analyses indicated that the two measures developed for the study, the Child Healthcare Experiences Inventory (CHEI) and the Healthcare Behavior Inventory (HBI) demonstrated good internal consistency and content validity. The primary analyses revealed that childhood healthcare experience, healthcare attitudes, and optimism each predicted current healthcare behavior. A significant interaction was found with optimism moderating the relation between childhood healthcare experience and current young adult healthcare behavior.

The relations between healthcare attitudes, optimism, and current healthcare behavior support the HBM model, which proposes that cognitive processes influence healthcare behavior. It might be that individuals who have had prior positive experience, hold positive healthcare attitudes, and are optimistic tend to perceive fewer barriers to healthcare or may be more likely 
to perceive more possible benefits from taking a particular healthcare action. However, the specific ways in which optimism and healthcare attitudes may impact healthcare behaviors, within the various domains of the HBM framework (e.g., perceived barriers), is unknown; more research is needed to further investigate these possible processes.

Although the three variables were related, healthcare attitudes were not found to moderate the relation between childhood healthcare experience and current healthcare behaviors. It is possible that the quality of childhood healthcare experience influences the development of healthcare attitudes in that individuals who experience more positive healthcare interactions are more likely to develop more positive healthcare attitudes. Conversely, individuals who possess more adaptive healthcare attitudes may be more likely to recall positive, as compared to negative, childhood healthcare experiences. Similar interpretations could extend to the relation between healthcare attitudes and healthcare behaviors. Regardless of the interpretation, it is important to appreciate that prior healthcare experience and current healthcare experience are linked to attitudes about healthcare. It was expected that, consistent with the HBM, healthcare experience and current healthcare behavior would be associated with attitudes towards healthcare. Until the directions of these relations are determined, healthcare professionals should not ignore a patient's prior experiences or attitudes about healthcare.

Optimism was not only found to be significantly associated with both prior and current healthcare behavior, it was also demonstrated to significantly moderate the relation between childhood healthcare experience and adolescent healthcare behaviors. Specifically, when prior medical experiences have been positive, optimism does little to enhance current healthcare behavior; however, when prior experiences were poor, optimism improves behavior. Thus, optimism might serve as a buffer for individuals with prior negative childhood healthcare 
experiences. Consistent with other work (Morrill et al., 1996; Taylor, Kemeny, Aspinwall, Schneider, \& Rodriguez, 1992), an optimistic style might influence healthcare behavior and/or impressions of healthcare behavior. In the current study optimism was positively associated with childhood healthcare experiences; this relation has not been previously investigated in the literature. One explanation of this finding may be that prior positive healthcare experiences influence the development of an optimistic style. It is equally possible that optimistic individuals perceive experiences as more positive, thus reinforcing their optimism and supporting the notion that "optimism is both motivated and motivating" (Peterson, 2000, pp. 45). This interpretation of optimism as a variable that is motivating is further supported in the synergistic relation it displayed with childhood healthcare experience in predicting healthcare behaviors. Various theories speculate whether optimism is part of human nature or a product of learning (for a review, see Peterson, 2000). More research is required to understand how healthcare experiences may shape optimism and how optimism may shape healthcare experiences. Based on the correlational findings of the study, intervention or prevention programs could screen individuals for optimism in order to anticipate their likely behavior in or perceptions of healthcare situations. With this knowledge, healthcare professionals can provide services to help individuals have more positive experiences, which in turn may have an impact on their behaviors.

Limitations with the study should be noted. First, due to the homogeneity of the sample it is not clear whether the present findings generalize to other populations. Given that all measures were self-report, method variance may account for some of the significant relations. Including other informants of behavior (e.g., parent report of participants' childhood healthcare experience) and different methods of assessment (e.g., observational, medical records) might prove beneficial to future research in the area. Other moderators or mediators linking childhood healthcare 
experience and current adolescent healthcare behavior might be explored. For example, parental experiences, perceptions, and behaviors might be important variables to consider. Moreover, longitudinal research is in order to fully appreciate relations between childhood healthcare experience and later adolescent healthcare behavior.

Results from the current study help establish the groundwork for predictors of adolescents' healthcare behavior. Given that adolescents/young adults are in a critical maturational phase in terms of selecting healthy or unhealthy habits that will continue into adulthood (Feldman, 1998; Martinelli, 1999), and that college students typically engage in a number of unhealthy behaviors (Grace, 1997), it is critical to continue to explore variables related to the health behaviors of this population. These results are consistent with the HBM, which hypothesizes that cognitive variables affect healthcare behaviors. Although causality cannot be determined, the links between adolescent healthcare experiences and their prior experiences, attitudes, and optimism suggest several avenues for intervention. For example, interventions aimed at improving children's healthcare experience might prove beneficial at that time as well as years later. In addition, measures of healthcare attitudes and optimism might serve as important markers for healthy or unhealthy adolescent healthcare behavior. Continued study of these and other variables are important in order to help promote lifelong healthy behavior. 


\section{References}

Ajzen, I., \& Fishbein, M. (1977). Attitude-behavior relations: A theoretical analysis and review of empirical research. Psychological Bulletin, 84, 888-918.

Bachanas, P. J., \& Roberts, M. C. (1995). Factors affecting children's attitudes toward healthcare and responses to stressful medical procedures. Journal of Pediatric Psychology, 20, 261275.

Baron, R. M., \& Kenny, D. A. (1986). The moderator-mediator variable distinction in social psychologically research: Conceptual, strategic, and statistical considerations. Journal of Personality and Social Psychology, 5, 1173-1182.

Becker, M. H. (1990). Theoretical models of adherence and strategies for improving adherence.

In: S. E. Shumaker \& E. B. Schron (Eds.) Handbook of health behavior change (pp. 5-43). New York, NY: Springer.

Becker, M. H. (1977). The Health Belief Model and prediction of dietary compliance: A field experiment. Journal of Health and Social Behavior, 18, 348-366.

Bush, P. J., \& Holmbeck, G. N. (1987). Children's attitudes about healthcare: Initial development of a questionnaire. Journal of Pediatric Psychology, 12, 429-443.

Feldman, R. (1998). Child Development. Upper Saddle River, NJ: Prentice Hall.

Grace, T. W. (1997). Health problems of college students. Journal of American College Health, 45, 243-251.

Hackworth, S. R., \& McMahon, R. J. (1991). Factors mediating children's healthcare attitudes. Journal of Pediatric Psychology, 16, 69-85.

Janz, N. K., \& Becker, M. H. (1984). The Health Belief Model: A decade later. Health Education Quarterly, 11, 1-47. 
Martinelli, A. M. (1999). An explanatory model of variables influencing health promotion behaviors in smoking and nonsmoking college students. Public Health Nursing, 16, 263 269.

Morrill, A. C., Ickovics, J. R., Golubchikov, V. V., \& Beren, S. E. (1996). Safer sex: Social and psychological predictors of behavioral maintenance and change among heterosexual women. Journal of Consulting and Clinical Psychology, 64, 819-828.

Pan American Health Organization. (2005). The Health Belief Model. In Youth: Choices and change (pp. 49 - 55). Washington, DC: Author.

Pate, J. T., Blount, R. L., Cohen, L. L., \& Smith, A. J. (1996). Childhood medical experience and temperament as predictors of adult functioning in medical situations. Children's Healthcare, 25, 281-298.

Peterson C. (2000). The future of optimism. American Psychologist, 55, 44-55.

Peterson, C., Ross, A., \& Tucker, V. C. (2002). Hospital emergency rooms and children's healthcare attitudes. Journal of Pediatric Psychology, 27, 281-291.

Reyna, V. F., \& Farley, F. (2006). Risk and rationality in adolescent decision making. Psychological Science in the Public Interest, 7, 1-44.

Sax, L. J. (1997). Health trends among college freshman. Journal of American College Health, 45, 252-262.

Scheier, M. F., \& Carver, C. S. (1992). Effects of optimism on psychological and physical well being: Theoretical overview and empirical update. Cognitive Therapy Research, 16, 201228. 
Segerstrom, S. C., Taylor, S. E., Kemeny, M. E., \& Fahey, J. L. (1998). Optimism is associated with mood, coping, and immune change in response to stress. Journal of Personality and Social Psychology, 74, 1646-1655.

Taylor, S. E., Kemeny, M. E., Aspinwall, L. G., Schneider, S. G., \& Rodriguez, H. M. (1992). Optimism, coping, psychological distress, and high-risk sexual behavior among men at risk for Acquired Immunodeficiency Syndrome (AIDS). Journal of Personality and Social Psychology, 63, 460-473.

Ware, J. E. (1976). Scales for measuring general health perceptions. Health Service Research,11, $396-415$. 
Table 1

Descriptive statistics for study measures

\begin{tabular}{lcc}
\hline Variable & Mean & SD \\
\hline Childhood healthcare experience (CHEI) & 1993.77 & 412.88 \\
Healthcare attitudes (HCAQ) & 57.82 & 10.76 \\
Optimism (LOT) & 19.47 & 5.39 \\
Current healthcare behavior (HBI) & 2739 & 405.4 \\
Prior health perception (HPQ - Prior Health subscale) & 10.80 & 3.10 \\
Current health perception (HPQ - Current Health subscale) & 32.69 & 6.39 \\
Health attitude (HPQ - Attitude subscale) & 6.85 & 2.20 \\
\hline
\end{tabular}

Table 2

Correlations among predictor, moderators, and dependent variables

\begin{tabular}{lccc}
\hline & $\begin{array}{l}\text { Childhood healthcare } \\
\text { experience }(\mathrm{CHEI})\end{array}$ & $\begin{array}{l}\text { Optimism } \\
\text { (LOT) }\end{array}$ & $\begin{array}{l}\text { Healthcare } \\
\text { attitudes (HCAQ) }\end{array}$ \\
\hline Optimism (LOT) & $.197^{*}$ & & \\
Healthcare attitudes (HCAQ) & $.391^{* *}$ & $.269^{* *}$ & $.433^{* *}$ \\
Current healthcare behavior (HBI) & $.733^{* *}$ & $.307^{* *}$ & .40 \\
\hline
\end{tabular}

$* p<.05 * * p<.01$

Table 3

Child healthcare experience and healthcare attitudes predicting current healthcare behavior

\begin{tabular}{lccccccc}
\hline Dependent Variables & $F$ & $p$ & $\Delta R^{2}$ & $\beta$ & Beta & $t$ & $p$ \\
\hline $\begin{array}{l}\text { Current healthcare behavior } \\
\text { Block 1: }\end{array}$ & 62.40 & .00 & & & & & \\
$\quad$ Childhood healthcare experience & & & 0.56 & 0.65 & 0.66 & 9.07 & $.000^{* * *}$ \\
Block 2: & 41.42 & .00 & & & & & \\
$\quad$ Childhood healthcare experience & & & 0.01 & 0.23 & 0.66 & 9.07 & $.02^{*}$ \\
$\quad$ Healthcare attitudes & & & 0.03 & .069 & -0.04 & 2.39 & $.03^{*}$ \\
\hline Current healthcare behavior & & & & & & & \\
Block 1: & 22.60 & .00 & 0.19 & & & & \\
$\quad$ Childhood healthcare experience & & & & 16.31 & .433 & 4.75 & $.000^{* * *}$ \\
$\quad$ Healthcare attitudes & & & & 6.51 & .17 & 2.37 & $.020^{*}$ \\
Block 2: & 62.40 & .00 & .38 & & & & \\
$\quad$ Childhood healthcare experience & & & & 0.63 & 0.72 & 11.29 & .000 \\
Healthcare attitudes & & & & 12.28 & 0.05 & 3.45 & .09 \\
Child med. experience X attitudes. & & & & -.92 & 0.24 & 3.2 & .34 \\
\hline
\end{tabular}

$* p<.05 . * * * p<.001$. 
Table 4

Child healthcare experience and optimism predicting current healthcare behavior

\begin{tabular}{lccccccc}
\hline Dependent Variables & $F$ & $p$ & $\Delta R^{2}$ & $\beta$ & Beta & $t$ & $p$ \\
\hline Current healthcare behavior & & & & & & & \\
Block 1: & 94.86 & .00 & & & & & \\
$\quad$ Childhood healthcare experience & & & 0.49 & 0.67 & 0.70 & 9.74 & $.000^{* * *}$ \\
Block 2: & 52.55 & .00 & & & & & \\
$\quad$ Childhood healthcare experience & & & 0.49 & 0.64 & 0.67 & 9.31 & $.000^{* * *}$ \\
$\quad$ Optimism & & & .03 & 12.87 & 0.17 & 2.39 & $.019^{*}$ \\
\hline Current healthcare behavior & & & & & & & \\
Block 1: & 63.55 & .00 & 0.57 & & & & \\
$\quad$ Childhood healthcare experience & & & & 0.68 & 0.67 & 10.30 & $.000^{* * *}$ \\
$\quad$ Optimism & & & & 13.21 & .07 & 2.22 & $.029 *$ \\
Block 2: & 44.50 & .00 & .02 & & & & \\
$\quad$ Childhood healthcare experience & & & & 0.71 & 0.72 & 10.58 & .000 \\
Optimism & & & & 11.42 & 0.15 & 2.21 & .03 \\
Child med. experience X optimism & & & & 0.21 & 0.12 & 1.82 & $.07 *$ \\
\hline
\end{tabular}

$* p<.05 . * * * p<.001$. 


\section{Figure Legend}

Figure 1. Hypothesized relations among childhood healthcare experience, healthcare attitudes, and current healthcare behavior

Figure 2. Hypothesized relations among childhood healthcare experience, optimism, and current healthcare behavior

Figure 3. The interaction between childhood healthcare experience and optimism predicting current healthcare behavior 

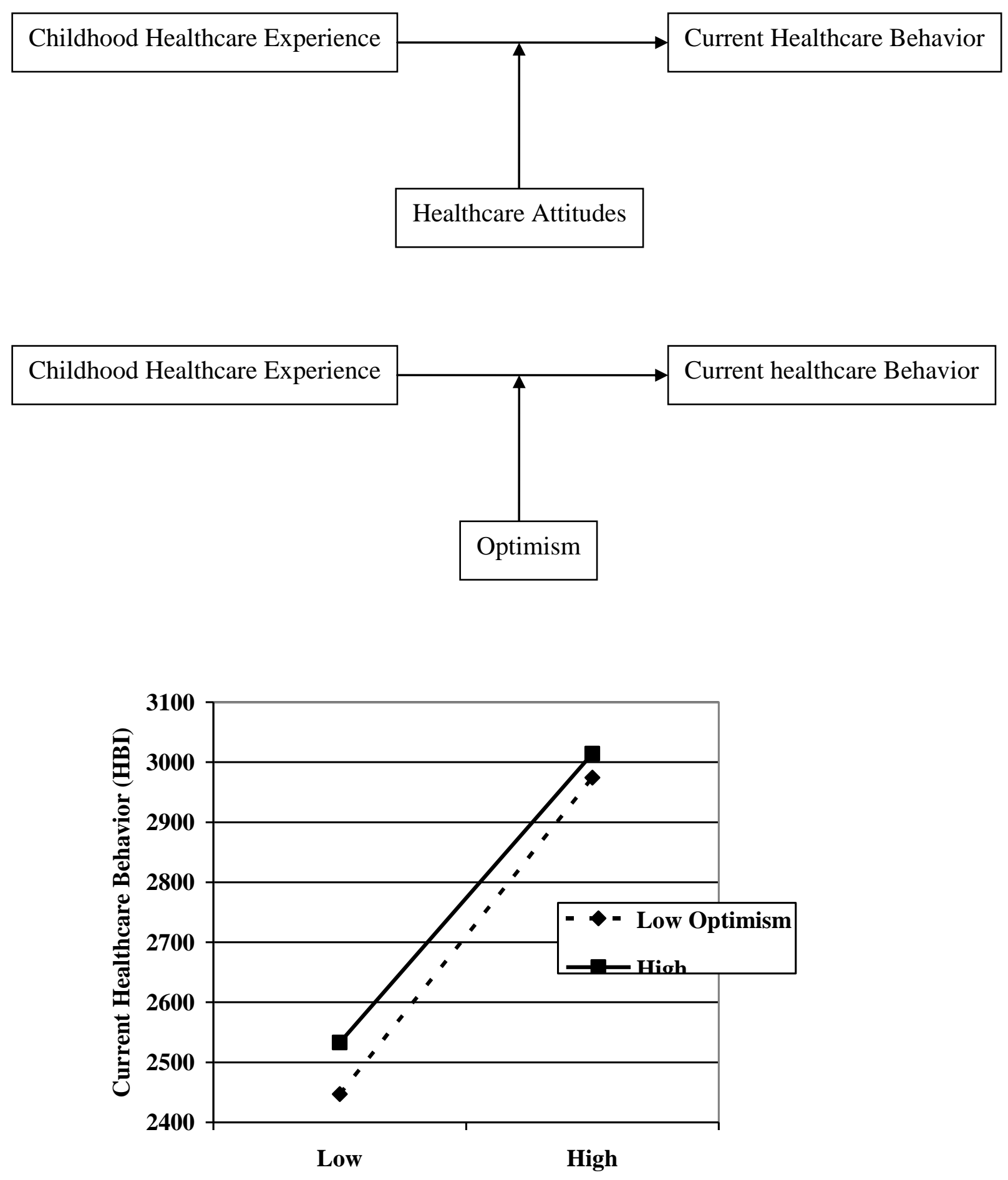

Childhood Healthcare Experiences (CHEI) 